Themed Issue: The Gut Microbiome and Immunity: How it is Shaped by Early Life

\title{
Early microbial contact, the breast milk microbiome and child health
}

\author{
S. Rautava* \\ Department of Pediatrics, University of Turku \& Turku University Hospital, Turku, Finland
}

\begin{abstract}
The significance of contact with microbes in early life for subsequent health has been the subject of intense research during the last 2 decades. Disturbances in the establishment of the indigenous intestinal microbiome caused by cesarean section delivery or antibiotic exposure in early life have been linked to the risk of immune-mediated and inflammatory conditions such as atopic disorders, inflammatory bowel disease and obesity later in life. Distinct microbial populations have recently been discovered at maternal sites including the amniotic cavity and breast milk, as well as meconium, which have previously been thought to be sterile. Our understanding of the impact of fetal microbial contact on health outcomes is still rudimentary. Breast milk is known to modulate immune and metabolic programming. The breast milk microbiome is hypothesized to guide infant gut colonization and is affected by maternal health status and mode of delivery. Immunomodulatory factors in breast milk interact with the maternal and infant gut microbiome and may mediate some of the health benefits associated with breastfeeding. The intimate connection between the mother and the fetus or the infant is a potential target for microbial therapeutic interventions aiming to support healthy microbial contact and protect against disease.
\end{abstract}

Received 1 April 2015; Revised 14 May 2015; Accepted 15 May 2015; First published online 8 June 2015

Keywords: breast milk, immune tolerance, intestinal microbiome

\section{Introduction}

Our understanding of the significance of microbes for human health has expanded over the last decades from viewing bacteria solely as potential pathogens to a dawning appreciation of the complexity of host-microbe interactions in health and disease. The early triumphs in clinical microbiology revealed the causative role of specific microbes in classical infectious diseases such as cholera, dysentery or diphtheria. Subsequent research has elucidated multifactorial causal networks involving potential pathogens, as well as a myriad of environmental and host susceptibility factors in the development of conditions also classified as infectious diseases such as community-acquired pneumonia or tuberculosis. More recently, we have begun to grasp the significance of environmental and indigenous microbes for host physiology and the development of noninfectious disease. Microbes have a profound effect on maintaining homeostasis in key physiological processes including immune, neural and metabolic functions. Perhaps even more importantly, intimate contact with the microbial world is necessary for normal development in early life.

\section{Microbes and host immune maturation}

Our current knowledge regarding the developmental significance of early microbial contact stems from experimental

*Address for correspondence: Dr S. Rautava, Specialist in Pediatrics, Neonatologist, Adjunct Professor in Experimental Pediatrics, Department of Pediatrics, University of Turku \& Turku University Hospital, Kiinamyllynkatu 4-8, 20520 Turku, Finland.

(Emailsamrau@utu.fi) studies using animals raised in controlled microbial environments the most revealing of which is total lack of microbial contact ('germ-free' animals). Hallmark animal studies have demonstrated that animals devoid of normal host-microbe interaction in early life exhibit defective maturation of the alimentary and immune systems and appear to be particularly prone to allergic-type adaptive immune responses. ${ }^{1,2}$ It is of particular note that introduction of a single bifidobacterium strain in the neonatal period is reportedly sufficient for inducing at least some degree of immune maturation. ${ }^{1}$ More recently, even intestinal contact with a specific microbial surface polysaccharide alone has been reported to restore a more physiological immune phenotype in germ-free mice. ${ }^{2} \mathrm{On}$ the other hand, certain disease states may be induced in experimental animals merely by colonizing them with a microbiome from an affected individual. In a beautiful series of experiments by Ridaura et $_{\mathrm{al}}{ }^{3}$ germ-free mice colonized with fecal microbes obtained from an obese human exhibited excessive weight and fat mass gain whereas mice whose gut microbiome originated from a lean individual remained lean. These animal studies clearly demonstrate that intestinal microbial colonization and contact with indigenous microbes in the neonatal period is crucial for normal immune and metabolic development.

An association between the composition of the intestinal microbiome and chronic non-infectious disease appears evident based on accumulating data from human studies. Aberrant gut colonization patterns have been reported in a wide variety of human diseases including atopic diseases, ${ }^{4-7}$ inflammatory bowel disease (IBD), ${ }^{8,9}$ necrotizing enterocolitis (NEC) in preterm infants, ${ }^{10,11}$ diabetes mellitus, ${ }^{12,13}$ obesity ${ }^{14,15}$ and 
neurological conditions ranging from autistic spectrum disorders ${ }^{16}$ to Parkinson's disease. ${ }^{17}$ The pathogenesis of most if not all of the chronic disorders in which indigenous microbes are thought to play a role involves immune-mediated or inflammatory processes and it is therefore plausible to hypothesize that pathological host-microbe interaction may have a causal role in their development.

Most reports showing a link between disease and gut microbiome composition are cross-sectional by design and therefore inherently incapable of establishing causality. Data from a limited number of longitudinal studies indicate, however, that the development of eczema ${ }^{6}$ and obesity ${ }^{15}$ in childhood may be preceded by aberrant gut colonization patterns in early infancy. Further corroboration for the hypothesized causal role for deviant early microbial contact in disease development has been provided by experimental animal models showing that the disease phenotype may be transferred with the gut microbiome at least in the case of obesity. ${ }^{3,18,19}$ Causality may also be indirectly inferred from epidemiological studies assessing the impact of factors known to perturb early gut colonization on later health.

\section{Mode of delivery}

Newborn infants acquire their indigenous microbiome primarily from the mother. It is well established that a substantial inoculum is provided by maternal intestinal and vaginal microbes during delivery and from an evolutionary point of view it is likely that this serves a specific purpose by guiding neonatal colonization. It is also well known that infants born by cesarean section delivery exhibit increased neonatal morbidity including respiratory problems and hypoglycemia compared with vaginally delivered infants. These issues arising from delayed adaptation to extrauterine life have been attributed to the lack of stress signals provided by the mother during vaginal delivery. Interestingly, birth by cesarean section is also associated with increased risk of chronic immune-mediated or inflammatory conditions such as asthma, ${ }^{20}$ type I diabetes ${ }^{21}$ and obesity. ${ }^{22,23}$ According to a recent Danish epidemiological study comprizing of 1.9 million subjects, the risk of asthma or IBD is $\sim 1$.2-fold in infants born by cesarean section and smaller but statistically significant increases in occurrence of juvenile arthritis, immune deficiencies and leukemia were also observed. $^{24}$ It is likely that the increased morbidity in infants born by cesarean section is at least partially attributable to altered early gut colonization. In line with this notion, the highest risk of obesity has been observed in individuals born by cesarean section and exposed to antibiotics, which also perturb microbial colonization. ${ }^{25}$ The early gut microbiome during the 1 st days of life in infants born vaginally shares features with the vaginal microbial community whereas in infants born by cesarean section the early gut microbiome resembles that of the maternal skin. ${ }^{26,27}$ The vaginal lactobacilli in the infant gut are rapidly replaced by microbes from other sources ${ }^{27}$ and most gut microbes in vaginally delivered infants originate in the maternal gut. The significance of delivery mode to intestinal colonization is demonstrated by the study by Jakobsson et al. ${ }^{28}$ according to which the intestinal microbial diversity is lower throughout the first 2 years of life in individuals delivered by cesarean section. The differences in the gut microbiome are still detectable between cesarean and vaginally delivered infants at the age of 7 years. ${ }^{29}$ These differences are also reflected in infant immune development up to the age of 12 months. ${ }^{30}$

\section{Early antibiotic exposure}

Exposure to antibiotics is known to exert a devastating effect on the gut microbiome. The intestinal microbial balance has been reported to recover after antibiotic exposure but there are also data to suggest that repeated antibiotic perturbation may result in incomplete microbial recovery. ${ }^{31-33}$ In the neonatal period, empirical antibiotic treatment has been associated with increased occurrence of $\mathrm{NEC}^{34}$ and late-onset sepsis ${ }^{35}$ and short-term gut microbiota perturbations including lower diversity $^{36}$ and particularly reduced colonization with bifidobacteria ${ }^{33,37}$ and bacteroides. ${ }^{38}$ Alarmingly, perinatal antibiotic exposure has been reported to result in long-term metabolic consequences and obesity in an experimental animal model even when the alterations in gut microbiota composition caused by antibiotics are reversible. ${ }^{19}$ Accumulating epidemiological data indicate that antibiotic perturbation of microbial contact during the critical developmental window in early life may have long-term health implications also in humans.

Early antibiotic use is associated with increased risk for a variety of chronic non-infectious diseases in later life. Antibiotic exposure during the 1st year of life is significantly more common in children who later develop IBD $^{39,40}$ and exposure to antibiotics during the first 6 months of life has been reported to be associated with an increase in body mass up to the age of 3 years. ${ }^{41}$ The risk of developing asthma at school age was independently increased by antibiotic exposure in the 1st week of life in a birth cohort study of $>5000$ children in Sweden. ${ }^{42}$ This association is plausible in the light of data indicating that neonatal antibiotic exposure results in decreased early gut microbiome diversity, ${ }^{36}$ which in turn is reportedly associated with the development of asthma in later life. ${ }^{7}$ It is of note that also maternal antibiotic use during pregnancy appears to be associated with increased risk of asthma in childhood. ${ }^{43}$ These epidemiological associations must be interpreted with caution since they may be explained by an underlying immunological factor predisposing to both infections and immune-mediated or inflammatory disease. The infection for which antibiotics had been administered may also play a role in disease pathogenesis. Nonetheless, careful examination of the available data reveals important time and dose-dependent effects, ${ }^{40}$ which may be interpreted to corroborate the hypothesis of long-term consequences as a result of microbial perturbation during a critical developmental period. 


\section{Microbial transfer from the mother}

Microbes constituting the indigenous human gut microbiome are not frequently found in the environment, which implies that infants acquire their gut microbes from other humans. As alluded to above, it is thought that the mother is the most important source of colonizing bacteria and, in line with this notion, infants have been shown to receive a significant bacterial inoculum during vaginal delivery. ${ }^{26,44}$ Less is known about other routes of microbial transfer.

\section{Microbial contact during fetal life}

It was long assumed that the fetus develops in a sterile environment without microbial contact except in cases of chorionic and fetal infection, which often lead to premature birth or fetal demise. Epidemiological and experimental studies have shown, however, that the maternal microbial environment has a significant impact on the risk of immune-mediated disease later in childhood. Douwes et al. ${ }^{45}$ have reported that children whose mothers have lived in a farming environment and hence presumably exposed to microbes more than their more urbandwelling counterparts, display a reduced risk for asthma. The investigators later isolated a bacterium, Acinetobacter lwoffi, from cow sheds and demonstrated that exposing pregnant mice to it protected the offspring from asthma in an experimental animal model. ${ }^{46}$ The precise mechanism of this indirect in utero microbial contact remains unknown. Interestingly, however, it is becoming evident that the healthy fetus may also be directly exposed to microbes.

The presence of microbes or their components in the uterus during pregnancy ${ }^{47}$ and also during the non-pregnant state ${ }^{48}$ appears to be a physiological phenomenon. Microbes or microbial DNA have been detected in the placenta ${ }^{49,50}$ and the umbilical cord ${ }^{51}$ in healthy pregnancy as well as in infant meconium, which formed during fetal life. ${ }^{10,26,52}$ It has recently been suggested that the human placenta harbors a distinct microbiome predominated by Proteobacteria. ${ }^{50}$ The origin and physiological role of the hypothesized in utero microbiome remains unknown. However, there are data to suggest that the placenta microbiome is modulated by excessive maternal weight gain during pregnancy. ${ }^{53}$ Microbes in the placenta have also been reported to be associated with placenta innate immune function. ${ }^{54}$ Cross analyses of separate data sets have suggested that the placenta microbiome shares features with the microbial community detected in the oral cavity, ${ }^{50}$ which is particularly interesting given the suggested association between poor dental health and complications of pregnancy including prematurity. ${ }^{55}$ On the other hand, experiments conducted in a murine model have shown that labeled bacteria introduced to the intestine of pregnant mice may be detected in the placenta and in meconium harvested after sterile cesarean section delivery, ${ }^{51,56}$ which suggests a link between the microbes in the maternal gut, the placenta and the fetal gut.

It should be born in mind that the discovery of the placenta microbiome was based on data obtained by sequencing of the
$16 \mathrm{~S}$ rRNA gene and therefore only demonstrates the presence of microbial DNA without direct evidence of viable bacteria. Nonetheless, the presence of microbial DNA in the intrauterine compartment suggests that the fetus may be in direct contact with microbial components during gestation. Meconium, which is formed during intrauterine life, also appears to harbor a unique microbial community. ${ }^{10,26,52}$ The meconium microbiome is rich in bacilli and other Firmicutes and drastically differs in composition from the gut microbiome later in the neonatal period. ${ }^{57}$ There is indirect evidence based on separate studies suggesting that the microbes in meconium originate from swallowed amniotic fluid. ${ }^{58}$ Interestingly, the composition of the meconium microbiome is not only dependent on maternal health status but also seems to be associated with subsequent child health. ${ }^{52}$

\section{Breast milk as modulator of the infant gut microbiome and source of microbes}

After birth, the most important determinant of infant gut colonization is breastfeeding. Breastfed infants harbor an intestinal microbiome dominated by Aktinobacteria and particularly bifidobacteria while the gut microbiome in formulafed infants is more diverse and resembles that of older children. ${ }^{59,60}$ The predominance of bifidobacteria in infant feces is thought to result from factors in breast milk, which favor the growth of these bacteria. A considerable amount of energy in breast milk is provided in the form of indigestible oligosaccharides, which do not provide energy to the infant but may be utilized by intestinal microbes. The functions of human milk oligosaccharides include acting as decoy receptors for potential pathogens and thus protection from infectious disease, and promoting the growth of bifidobacteria (reviewed by Bode $\left.^{61}\right)$. Bifidobacteria in infant feces are often considered to be 'good' or 'health-promoting' and, indeed, colonization with bifidobacteria has been associated with reduced risk for both allergic disease ${ }^{5}$ and excessive weight gain. ${ }^{15,62}$ It is not clear to what extent the documented beneficial long-term health effects of breast milk including reducing the risk of obesity and immune-mediated disease (reviewed by Rautava and Walker ${ }^{63}$ ) are mediated via modulation of the gut microbiome. It is important to recognize that the composition of breast milk varies between mothers, which may at least partially explain the discrepant data concerning the relationship between breastfeeding and the risk of immune-mediated disease such as allergy. Furthermore, the mechanisms underlying the association between abundance of intestinal bifidobacteria and favorable health outcomes still remain unknown.

In addition to specifically promoting the growth of intestinal microbes thought to be beneficial to health, recent evidence indicates that breast milk may be a source of bacteria for the infant gut. Even non-lactating mammary gland tissue has been reported to contain both live bacteria and bacterial DNA. ${ }^{64}$ The microbial community in breast milk characterized by $16 \mathrm{~S}$ rRNA gene pyrosequencing appears to be unique with a 
composition clearly distinct from that observed at other mucosal sites including the skin, gut, mouth or vagina. ${ }^{65}$ The origin of the bacteria in breast milk is currently not known but there are circumstantial evidence suggesting a link between the maternal gut and mammary gland. Increased intestinal bacterial translocation has been reported during pregnancy and lactation in experimental animals. ${ }^{66}$ Maternal intestinal microbes have been detected in immune cells circulating in peripheral blood and in breast milk in both mice and humans suggesting active and selective transport. ${ }^{66}$ In a clinical trial, the probiotic bacterium Lactobacillus reuteri has been detected in breast milk after oral intake in lactating mothers. ${ }^{67}$

The teleological reason for microbes in breast milk and their role in infant gut colonization remain largely speculative. The same bifidobacterium species have been consistently found in maternal feces, breast milk and the infant gut but the study provided no mechanistic insight as to whether the bifidobacteria in infant gut originated from breast milk or were transferred from the mother via other routes. ${ }^{68}$ Martin et al. ${ }^{69}$ studied breast milk, mammary areola and breast skin samples from eight breastfeeding mothers and oral swab and fecal samples from their infants. Identical Lactobacillus specimens were discovered from the breast milk, infant oral mucosa and infant feces in all mother-infant pairs. These data provide corroboration for the notion of bacterial transfer via breast milk but its significance for infant gut colonization is still unknown and it is likely that maternal and environmental transfer of bacteria takes primarily place by other routes.

The composition of the breast milk microbiome is affected by maternal factors. A significant change in the composition of the breast milk microbiome has been observed over the span of lactation. ${ }^{65}$ The most common genera in colostrum samples detected by $16 \mathrm{~S}$ sequencing included Leuconostoc, Weissella, Staphylococcus, Streptococcus and Lactococcus according to analyses of breast milk samples from 18 mothers from Finland. ${ }^{65} \mathrm{~A}$ significant increase in Veillonella, Prevotella and Leptotrichia was seen 1 and 6 months after delivery. In a similar study conducted using quantitative polymerase chain reaction (qPCR) to identify bacteria, Lactobacillus, Enterococcus and Streptococcus were the most prominent bacteria in breast milk and the abundance of Bifidobacterium and Enterococcus species increased throughout the lactation period. ${ }^{70}$ In breast milk samples collected from African mothers and analyzed by qPCR, streptococci, staphylococci and lactobacilli were the most prevalent bacteria but the breast milk microbiome composition varied as a function of maternal HIV status. ${ }^{71}$ The duration of gestation also seems to have an impact on the breast milk microbiome. Particularly the abundance of bifidobacterium species has been observed to be higher in breast milk from mothers who delivered at full term as compared with mothers of preterm infants. ${ }^{70}$ The significance of these differences remains to be determined.

Factors known to influence child health outcomes have also been reported to have an impact on the breast milk microbiome. As discussed above, delivery by cesarean section results in aberrant infant gut colonization and immune development and is associated with increased risk of obesity and chronic immune-mediated disease later in childhood. Interestingly, breast milk from mothers who had undergone cesarean section delivery exhibited a higher microbial diversity but reduced frequency of bifidobacteria according to a report based on milk samples obtained from 32 healthy mothers and analyzed by qPCR. $^{70}$ Whether the reduced bifidobacterium content in breast milk contributes to the decreased colonization by bifidobacteria observed in infants born by cesarean section is unknown. The mechanisms by which mode of delivery affects the breast milk microbiome, also remain to be determined but the changes are detectable at least until 6 months after delivery. ${ }^{65}$ The contribution of intrapartum antibiotic prophylaxis often administered to mothers before cesarean section delivery to altered breast milk microbiome is not known, nor is the effect of antibiotics administered during vaginal delivery. Interestingly, however, the observed qualitative and quantitative differences in breast milk microbial communities appear to be more pronounced in women undergoing elective cesarean section while the impact of surgical delivery in women in labor is more moderate. ${ }^{65}$ This has been interpreted to suggest that the stress and/or hormonal signals related to labor have an impact on bacterial transfer to the mammary gland. ${ }^{65}$

The associations between breastfeeding and childhood obesity $^{72}$ on the one hand and early gut microbiome composition and weight gain ${ }^{15}$ on the other set an interesting background to the observation according to which differences in the microbial composition of breast milk are detectable between overweight or normal weight mothers. ${ }^{65}$ Obese mothers exhibited higher total bacterial counts and particularly higher counts of staphylococci and lactobacilli in their breast milk while the number of bifidobacteria was higher in the breast milk of normal weight mothers. Changes related to excessive weight gain during pregnancy were similar to those seen in obese mothers. Whether the breast milk microbiome provides an explanatory link between breastfeeding duration, the gut microbiome and obesity is currently not understood. Interestingly, maternal overweight is also associated with decreased concentration of immunomodulatory factors in breast milk. ${ }^{73}$

\section{The immunomodulatory potential of breast milk}

Breast milk contains a vast array of immune factors such as immunoglobulins, oligosaccharides, innate immune receptors and antimicrobial proteins including lysozyme, lactoferrin and defensins, which provide protection against infectious disease during the vulnerable 1st months of life (reviewed by Rautava and $\mathrm{Walker}^{63}$ ). While the well-established protective effects of breastfeeding against early infections are likely to be mostly attributable to this passive immunoprotection (and probably also reduced exposure to potentially contaminated water), the role of breast milk components with active immunomodulatory potential remains less well elucidated. It is likely that the reduction in the occurrence of NEC associated with human 
milk feeding as compared with cow's milk-based formula documented in preterm infants ${ }^{74}$ is mediated by both breast milk antimicrobial proteins ${ }^{75}$ and the immunomodulatory and maturational properties of breast milk, ${ }^{76,77}$ as well as the beneficial effects of breast milk on gut microbiota discussed above. Recently, oropharyngeal administration of minute amounts of colostrum has been shown to induce the production of secretory IgA antibodies and lactoferrin and reduce the incidence of clinical sepsis in extremely premature infants. ${ }^{78}$ The intimate interplay between intestinal and breast milk microbes and the immunomodulatory factors in breast milk may also at least partially explain the long-term protective effects of breastfeeding against immune-mediated and inflammatory chronic disease.

\section{Breast milk as a vehicle for immune tolerance induction}

Transforming growth factor (TGF) $\beta$, one of the most thoroughly investigated immunomodulatory factors in breast milk, may be considered a paradigmatic mediator of host-microbe interaction in breast milk and the infant gut. The functions of TGF- $\beta$ range from inducing the production of IgA class antibodies and direct anti-inflammatory and maturational effects on immune and intestinal epithelial cells to induction of regulatory $\mathrm{T}$ cells central to establishing and maintaining immune tolerance (reviewed by Rautava and Walker ${ }^{63}$ ). Of the three isoforms of TGF- $\beta$, TGF- $\beta 2$ is the most abundant in breast milk while TGF- $\beta 1$ is produced by immune cells in the intestine. Data from experimental animal models suggest that breast milk may be an important source of TGF- $\beta$ to the developing intestine in early life when endogenous production of TGF- $\beta$ by the developing immune system is low. ${ }^{79}$ Breastfed infants have been observed to exhibit higher serum TGF- $\beta 2$ concentrations throughout the 1 st year of life when compared with formula-fed infants suggesting that factors in breast milk may also act as triggers for TGF- $\beta$ production in the infant. ${ }^{80}$

TGF- $\beta$ is a key mediator of active immune tolerance (Box 1). ${ }^{81}$ Studies conducted in a murine asthma model have elegantly demonstrated that TGF- $\beta$ is necessary for induction of immune tolerance toward environmental antigens via breast milk. ${ }^{82}$ Recently published data indicate that TGF- $\beta 2$ regulates intestinal epithelial cell inflammatory responses against bacterial antigens. ${ }^{83}$ In clinical studies, breast milk TGF- $\beta$ concentrations have been found to be inversely associated with the risk of eczema and allergy-related outcomes in the child. ${ }^{84,85}$ The breast milk concentration of TGF- $\beta 2$ is reportedly lower in atopic than healthy mothers, ${ }^{86}$ and the contributions of hereditary risk and breast milk factors to the risk of disease in the offspring are not clear. Interestingly, however, there are data suggesting that breast milk TGF- $\beta$ may also play a role in establishing tolerance toward indigenous microbes.

NEC is a devastating intestinal inflammatory condition, which afflicts primarily preterm neonates. The pathogenesis of NEC is complex and still largely unknown, but intestinal and immunologic immaturity and increased intestinal bacterial translocation are thought to result in an excessive inflammatory reaction against intestinal microbes leading to a vicious cycle of yet more impaired gut barrier and propagated inflammation (reviewed by Neu and Walker ${ }^{87}$ ). It has recently been reported that preterm infants who develop NEC have lower serum TGF- $\beta$ concentrations from birth. ${ }^{88}$ The protective effect of human breast milk against the development of NEC has been convincingly demonstrated in clinical trials ${ }^{74}$ but the mechanisms underlying the clinical efficacy are not fully established. Experimental studies using immature human gut models indicate that exposure to TGF- $\beta 2$ in a concentration corresponding to that in breast milk exerts a direct antiinflammatory effect in the immature intestine ${ }^{76}$ and induces maturation in intestinal epithelial cells. ${ }^{77}$ Furthermore, TGF$\beta 2$ modulates intestinal epithelial cell innate immune responses to bacterial antigens and restores potentially detrimental stress responses elicit against lipopolysaccharide, a cell well component of gram-negative microbes. ${ }^{83}$ These effects are reflected in the results of a clinical study, which suggest that TGF- $\beta 2$ concentrations in the human milk received by very low birth weight preterm infants tend to correlate inversely with the incidence of NEC and feeding intolerance. ${ }^{89}$ More insight into the effects of breast milk TGF- $\beta$ in inducing tolerance toward microbes and protection against intestinal inflammation is provided by a series of experiments conducted in a porcine model. Piglets fed colostrum rich in TGF- $\beta 2$ exhibited significantly lower intestinal inflammation when compared with those fed infant formula with a low concentration of TGF- $\beta 2$ and TGF- $\beta 2$ directly attenuated inflammatory responses toward bacterial antigens in porcine intestinal epithelial cells. ${ }^{90}$ In a parallel manner, infant formula supplemented with TGF$\beta 2$ has been reported to attenuate intestinal inflammation in an experimental murine IBD model. ${ }^{91}$

In addition to breast milk TGF- $\beta 2$ modulating inflammatory responses elicited against microbial antigens, both the concentration of TGF- $\beta 2$ in breast milk and neonatal responsiveness to TGF- $\beta 2$ seem to be modulated by specific microbes. Maternal supplementation with the probiotic bacterium Lactobacillus rhamnosus GG has been shown to significantly increase the amount of TGF- $\beta 2$ in breast milk. ${ }^{92}$ A similar increase has subsequently been reported after maternal supplementation with the combination of $L$. rhamnosus GG and Bifidobacterium lactis $\mathrm{Bb}-12,{ }^{93}$ while consumption of $L$. reuteri is associated with decreased breast milk TGF- $\beta 2$ content. ${ }^{94}$ The expression of SMAD7, a key negative regulator of TGF- $\beta$ responsiveness, has been reported to be decreased in preterm infants after supplementation with the probiotic Bifidobacterium breve. ${ }^{95}$ Given the interactions between microbes in the maternal gut, breast milk and the fetal gut on the one hand and TGF- $\beta$ expression and function on the other, it is intriguing to speculate that the function of microbes and microbial components in breast milk might be to introduce them to the infant gut in a tolerogenic immune milieu created by factors including TGF- $\beta$ to induce tolerance to colonizing maternal microbes. 


\section{Modulating child health outcomes through specific probiotic interventions on the pregnant and breastfeeding mother}

After the significance of early microbial contact for the development of immune-mediated and inflammatory conditions was realized, considerable research interest has been focused on modulating host-microbe interactions during the vulnerable perinatal period and early infancy. Promotion of breastfeeding, prudent use of antibiotics and reassessing the benefits and potential harms of cesarean section deliveries are obvious and presumably effective means to reduce the burden of chronic disease on the population level. In addition, supplementation with specific microbes with desirable effects on the host offers itself as a means of directly influencing early host-microbe interaction. Probiotics have recently been defined as 'live microorganisms that, when administered in adequate amounts, confer a health benefit on the host (Box 1). 96 Specific probiotics have been extensively studied and shown efficacy in reducing the risk of atopic disease and eczema. ${ }^{97}$ There are a number of clinical trials suggesting that probiotic bacteria may reduce the incidence of NEC in preterm infants and the authors of a recent meta-analysis of clinical studies conclude the probiotics are efficacious in prevention of NEC. ${ }^{98}$ However, probiotic effects are highly strain and species-specific in vitro and in vivo and it may not be appropriate to devise metaanalyses based on studies using different probiotic bacteria. A sufficiently powered clinical trial assessing the effect of specific probiotics on the incidence of NEC is therefore called for. ${ }^{99}$ Specific probiotics have also been suggested to be of potential benefit in prevention or treatment of various immune-mediated or inflammatory conditions ranging from obesity and the metabolic syndrome to IBD and even certain neurological conditions. It is often assumed that probiotics exert their effects by modulating the composition of the gut microbiome. Interestingly, however, recent research advances indicate that specific probiotics may offer health benefits to the child indirectly via the pregnant or breastfeeding mother.

The first clinical trial assessing the impact of maternal probiotic supplementation on infant health was conducted in Finland. ${ }^{100}$ The primary objective of the study was to reduce the occurrence of eczema in children at high risk due to family history of atopic disease. Maternal supplementation with the probiotic L. rhamnosus GG or placebo was commenced in 159 mothers 2-4 weeks before delivery and continued 6 months after delivery in a randomized, double-blind design. If breastfeeding was discontinued during this time period, the study product was given directly to the infant. The risk of developing eczema was significantly reduced by the probiotic intervention at the age of 2 years and the protective effect persisted to the ages of 4 and 7 years. ${ }^{101,102}$ Perhaps even more interestingly, a similar or more pronounced protective effect was seen in the subgroup of infants who received the probiotic intervention solely via the pregnant and breastfeeding mother, ${ }^{92}$ while little or no impact on infant gut microbiome composition and only transient colonization with L. rhamnosus GG was observed to result from the probiotic intervention. ${ }^{103,104}$

The efficacy of maternal probiotic supplementation in prevention of eczema in high-risk infants was later confirmed in a randomized, placebo-controlled trial of 241 mother-infant pairs in whom maternal supplementation with either the probiotic combination of L. rhamnosus LPR and Bifidobacterium longum BL999 or L. paracasei ST11 and B. longum BL999 was commenced 2 months before delivery and continued for 2 months after birth during exclusive breastfeeding while none of the infants received probiotics directly. ${ }^{105}$ A highly significant reduction in the risk of developing eczema was seen in both probiotic groups compared with placebo at the age of 2 years. In line with the results of these clinical trials, a recent meta-analysis of probiotic studies with different administration protocols concluded that both prenatal and postnatal probiotic intervention is needed to achieve reduction in eczema in the child. ${ }^{97}$ As discussed above, compiling data obtained from studies using different probiotic strains may not be appropriate. It is likely that even when effective, only certain probiotic bacteria elicit a beneficial effect against specific disease states while other probiotic strain may be ineffective or even have a detrimental effect. In addition, the mechanisms of the protective effects of maternal probiotic administration remain poorly elucidated.

There are data to suggest that maternal intervention with specific probiotics during pregnancy may modulate innate immune function in the placenta and in the fetal gut. ${ }^{54}$ Experimental studies have shown that fetal human gut models are responsive to bacteria and that the probiotic L. rhamnosus GG and its components exert an immunomodulatory effect in the human fetal intestinal epithelium. ${ }^{106}$ Still, it is not known whether particular probiotic bacteria administered to the mother are transferred to the amniotic cavity or the fetus despite experimental data suggesting that specific microbes introduced in the maternal gut during pregnancy may be recovered in the placenta in mice. ${ }^{51}$ After birth, there are convincing experimental and clinical data indicating that maternal gut bacteria are transferred to breast milk. ${ }^{66}$ Specifically, the probiotic $L$. reuteri has been detected in breast milk after oral administration in lactating women. ${ }^{67}$ These data suggest that the breastfeeding infant may receive the probiotic via breast milk. In addition, the protective effects of defined probiotic interventions during breastfeeding may be partially explained by modulation of breast milk immune factors including TGF- $\beta$ as discussed above.

\section{Conclusions}

Realization of the profound impact early-life microbial contact may have on human health has lead to a rapid increase in our understanding of factors which may disturb or support the establishment of the indigenous intestinal microbiome (Fig. 1). The impact of cesarean section delivery on the risk of developing immune-mediated and inflammatory conditions are 


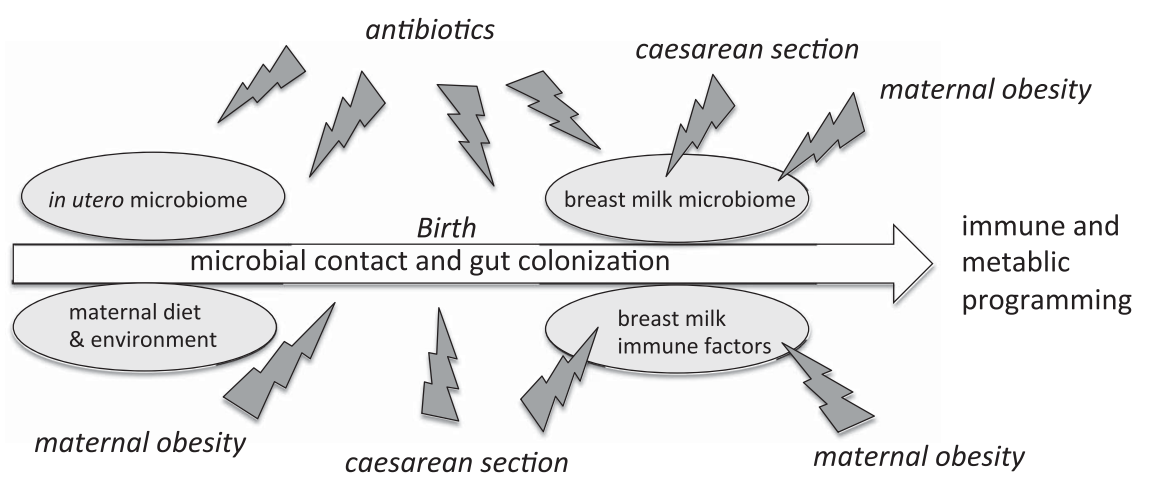

Fig. 1. Factors modulating early microbial contact and intestinal colonization, which guide immune maturation and thus affect the risk of developing chronic disease. Major threats to healthy host-microbe interaction include maternal obesity, cesarean section delivery and exposure to antibiotics during the prenatal and perinatal periods and early infancy.

\section{Box 1. Key concepts.}

Microbiota: the community of microorganisms residing in a distinct ecological niche or environment.

Microbiome: the genetic pool of the microbiota of a given location and their interaction with the environment.

N.B. the terms 'microbiota' and 'microbiome' are often used interchangeably as if they were synonymous.

Microbial contact: direct interaction with a microbe or microbial component that results in a host response (e.g. inflammation or tolerance).

Immune tolerance: any mechanism by which a potentially injurious immune response is prevented, suppressed or shifted to a non-injurious immune response. ${ }^{81}$

Probiotics - live microorganisms that, when administered in adequate amounts, confer a health benefit on the host. ${ }^{96}$

convincingly documented by epidemiological studies and backed by microbiological and experimental data. In a similar fashion, early antibiotic exposure has been shown to have detrimental long-term consequences. These risks should be taken into consideration in clinical care and particularly when devizing practice guidelines on the population level.

Investigations on microbial contact during fetal life and its significance for health are a new field of study, which may influence both our notion of developmental origins of disease and future care of the pregnant mother. After birth, the beneficial effects of breastfeeding are well known but further complemented with regard to both scope and mechanisms by the recent discovery of the breast milk microbiome and its connections with immune factors in breast milk.

Finally, the pregnant and breastfeeding mother is an emerging target for interventions aiming to support healthy microbial contact and to reduce the risk of chronic disease in the offspring.
Maternal interventions with specific probiotics have proven to be efficacious in reducing the risk of eczema. No serious adverse effects have been reported in clinical trails but the long-term efficacy and safety of probiotic interventions need to be monitored. In the future, research efforts should be aimed to find optimal means to combat the epidemics of obesity and immunemediated disease.

\section{Acknowledgments}

Professors Erika Isolauri and Seppo Salminen are acknowledged for valuable and insightful comments regarding the manuscript.

\section{Financial Support}

The author is funded by the Emil Aaltonen Foundation and the Collegium for Science and Medicine of the University of Turku, Finland.

\section{Conflict of Interest}

None.

\section{References}

1. Sudo N, Sawamura S, Tanaka K, et al. The requirement of intestinal bacterial flora for the development of an $\operatorname{IgE}$ production system fully susceptible to oral tolerance induction. J Immunol. 1997; 159, 1739-1745.

2. Mazmanian SK, Liu CH, Tzianabos AO, Kasper DL. An immunomodulatory molecule of symbiotic bacteria directs maturation of the host immune system. Cell. 2005; 122, 107-118.

3. Ridaura VK, Faith JJ, Rey FE, et al. Gut microbiota from twins discordant for obesity modulate metabolism in mice. Science. 2013; 341, 1241214.

4. Kalliomäki M, Kirjavainen P, Eerola E, et al. Distinct patterns of neonatal gut microflora in infants in whom atopy was and was not developing. J Allergy Clin Immunol. 2001; 107, 129-134.

5. Björkstén B, Sepp E, Julge K, Voor T, Mikelsaar M. Allergy development and the intestinal microflora during the first year of life. J Allergy Clin Immunol. 2001; 108, 516-520. 
6. Abrahamsson TR, Jakobsson HE, Andersson AF, et al. Low diversity of the gut microbiota in infants with atopic eczema. J Allergy Clin Immunol. 2012; 129, 434-440.

7. Abrahamsson TR, Jakobsson HE, Andersson AF, et al. Low gut microbiota diversity in early infancy precedes asthma at school age. Clin Exp Allergy. 2014; 44, 842-850.

8. Schwiertz A, Jacobi M, Frick JS, et al. Microbiota in pediatric inflammatory bowel disease. J Pediatr. 2010; 157, 240-244.

9. Gevers D, Kugathasan S, Denson LA, et al. The treatment-naive microbiome in new-onset Crohn's disease. Cell Host Microbe. 2014; 15, 382-392.

10. Mshvildadze M, Neu J, Shuster J, et al. Intestinal microbial ecology in premature infants assessed with non-culture-based techniques. J Pediatr. 2010; 156, 20-25.

11. Mai V, Young CM, Ukhanova M, et al. Fecal microbiota in premature infants prior to necrotizing enterocolitis. PLoS One. 2011; 6, e20647.

12. Dunne JL, Triplett EW, Gevers D, et al. The intestinal microbiome in type 1 diabetes. Clin Exp Immunol. 2014; 177, 30-37.

13. Kostic AD, Gevers D, Siljander H, et al. The dynamics of the human infant gut microbiome in development and in progression toward type 1 diabetes. Cell Host Microbe. 2015; 17, 260-273.

14. Turnbaugh PJ, Hamady M, Yatsunenko T, et al. A core gut microbiome in obese and lean twins. Nature. 2009; 457, 480-484.

15. Kalliomäki M, Collado MC, Salminen S, et al. Early differences in fecal microbiota composition in children may predict overweight. Am J Clin Nutr. 2008; 87, 534-538.

16. De Angelis M, Piccolo M, Vannini L, et al. Fecal microbiota and metabolome of children with autism and pervasive developmental disorder not otherwise specified. PLoS One. 2013; 8, e76993.

17. Scheperjans F, Aho V, Pereira PA, et al. Gut microbiota are related to Parkinson's disease and clinical phenotype. Mov Disord. 2015; 30, 350-358.

18. Koren O, Goodrich JK, Cullender TC, et al. Host remodeling of the gut microbiome and metabolic changes during pregnancy. Cell. 2012; 150, 470-480.

19. Cox LM, Yamanishi S, Sohn J, et al. Altering the intestinal microbiota during a critical developmental window has lasting metabolic consequences. Cell. 2014; 158, 705-721.

20. Thavagnanam S, Fleming J, Bromley A, Shields MD, Cardwell CR. A meta-analysis of the association between caesarean section and childhood asthma. Clin Exp Allergy. 2008; 38, 629-633.

21. Cardwell CR, Stene LC, Joner G, et al. Caesarean section is associated with an increased risk of childhood-onset type 1 diabetes mellitus: a meta-analysis of observational studies. Diabetologia. 2008; 51, 726-735.

22. Barros FC, Matijasevich A, Hallal PC, et al. Cesarean section and risk of obesity in childhood, adolescence, and early adulthood: evidence from 3 Brazilian birth cohorts. Am J Clin Nutr. 2012; 95, 465-470.

23. Darmasseelane K, Hyde MJ, Santhakumaran S, Gale C, Modi N. Mode of delivery and offspring body mass index, overweight and obesity in adult life: a systematic review and meta-analysis. PLoS One. 2014; 9, e87896.

24. Sevelsted A, Stokholm J, Bønnelykke K, Bisgaard H. Cesarean section and chronic immune disorders. Pediatrics. 2015; 135, e92-e98.

25. Mueller NT, Whyatt R, Hoepner L, et al. Prenatal exposure to antibiotics, cesarean section and risk of childhood obesity. Int $J$ Obes (Lond). 2015; 39, 665-670.
26. Dominguez-Bello MG, Costello EK, Contreras M, et al. Delivery mode shapes the acquisition and structure of the initial microbiota across multiple body habitats in newborns. Proc Natl Acad Sci USA. 2010; 107, 11971-11975.

27. Matsumiya Y, Kato N, Watanabe K, Kato H. Molecular epidemiological study of vertical transmission of vaginal Lactobacillus species from mothers to newborn infants in Japanese, by arbitrarily primed polymerase chain reaction. J Infect Chemother. 2002; 8, 43-49.

28. Jakobsson HE, Abrahamsson TR, Jenmalm MC, et al. Decreased gut microbiota diversity, delayed bacteroidetes colonisation and reduced Th1 responses in infants delivered by caesarean section. Gut 2014; 63, 559-566.

29. Salminen S, Gibson GR, McCartney AL, Isolauri E. Influence of mode of delivery on gut microbiota composition in seven year old children. Gut. 2004; 53, 1388-1389.

30. Huurre A, Kalliomäki M, Rautava S, et al. Mode of delivery effects on gut microbiota and humoral immunity. Neonatology. 2008; 93, 236-240.

31. Dethlefsen L, Huse S, Sogin ML, Relman DA. The pervasive effects of an antibiotic on the human gut microbiota, as revealed by deep 16S rRNA sequencing. PLoS Biol. 2008; 6, e280.

32. Dethlefsen L, Relman DA. Incomplete recovery and individualized responses of the human distal gut microbiota to repeated antibiotic perturbation. Proc Natl Acad Sci USA. 2011; 108(Suppl. 1), 4554-4561.

33. Fouhy F, Guinane CM, Hussey S, et al. High-throughput sequencing reveals the incomplete, short-term recovery of infant gut microbiota following parenteral antibiotic treatment with ampicillin and gentamicin. Antimicrob Agents Chemother. 2012; 56, 5811-5820.

34. Cotten CM, Taylor S, Stoll B, et al. Prolonged duration of initial empirical antibiotic treatment is associated with increased rates of necrotizing enterocolitis and death for extremely low birth weight infants. Pediatrics. 2009; 123, 58-66.

35. Shah P, Nathan E, Doherty D, Patole S. Prolonged exposure to antibiotics and its associations in extremely preterm neonates the Western Australian experience. J Matern Fetal Neonatal Med. 2013; 26, 1710-1714.

36. Greenwood C, Morrow AL, Lagomarcino AJ. Early empiric antibiotic use in preterm infants is associated with lower bacterial diversity and higher relative abundance of Enterobacter. J Pediatrics. 2014; 165, 23-29.

37. Tanaka S, Kobayashi T, Songjinda P, et al. Influence of antibiotic exposure in the early postnatal period on the development of intestinal microbiota. FEMS Immunol Med Microbiol. 2009; $56,80-87$.

38. Arboleya S, Sánchez B, Milani C, et al. Intestinal microbiota development in preterm neonates and effect of perinatal antibiotics. J Pediatrics. 2015; 166, 538-544.

39. Shaw SY, Blanchard JF, Bernstein CN. Association between the use of antibiotics in the first year of life and pediatric inflammatory bowel disease. Am J Gastroenterol. 2010; 105, 2687-2692.

40. Virta L, Auvinen A, Helenius H, Huovinen P, Kolho KL. Association of repeated exposure to antibiotics with the development of pediatric Crohn's disease - a nationwide, registerbased Finnish case-control study. Am J Epidemiol. 2012; 175, 775-784. 
41. Trasande L, Blustein J, Liu M, et al. Infant antibiotic exposures and early-life body mass. Int J Obes (Lond). 2013; 37, 16-23.

42. Goksör E, Alm B, Pettersson R, et al. Early fish introduction and neonatal antibiotics affect the risk of asthma into school age. Pediatr Allergy Immunol. 2013; 24, 339-344.

43. Stensballe LG, Simonsen J, Jensen SM, Bønnelykke K, Bisgaard H. Use of antibiotics during pregnancy increases the risk of asthma in early childhood. J Pediatr. 2013; 162, 832-838.

44. Makino H, Kushiro A, Ishikawa E, et al. Mother-to-infant transmission of intestinal bifidobacterial strains has an impact on the early development of vaginally delivered infant's microbiota. PLoS One. 2013; 8, e78331.

45. Douwes J, Cheng S, Travier N, et al. Farm exposure in utero may protect against asthma, hay fever and eczema. Eur Respir J. 2008; 32, 603-611.

46. Conrad ML, Ferstl R, Teich R, et al. Maternal TLR signaling is required for prenatal asthma protection by the nonpathogenic microbe Acinetobacter lwoffii F78. J Exp Med. 2009; 206, 2869-2877.

47. Stout MJ, Conlon B, Landeau M, et al. Identification of intracellular bacteria in the basal plate of the human placenta in term and preterm gestations. Am J Obstet Gynecol. 2013; 208, e1-e7.

48. Andrews WW, Goldenberg RL, Hauth JC, et al. Endometrial microbial colonization and plasma cell endometritis after spontaneous or indicated preterm versus term delivery. Am J Obstet Gynecol. 2005; 193, 739-745.

49. Satokari R, Grönroos T, Laitinen K, Isolauri E, Salminen S. Bifidobacterium and Lactobacillus DNA in the human placenta. Lett Appl Microbiol. 2009; 48, 8-12.

50. Aagaard K, Ma J, Antony KM, et al. The placenta harbors a unique microbiome. Sci Transl Med. 2014; 6, $237 \mathrm{ra65.}$

51. Jiménez E, Fernández L, Marin ML, et al. Isolation of commensal bacteria from umbilical cord of healthy neonates born by cesarean section. Curr Microbiol 2005; 51, 270-274.

52. Gosalbes MJ, Llop S, Vallés Y, et al. Meconium microbiota types dominated by lactic acid or enteric bacteria are differentially associated with maternal eczema and respiratory problems in infants. Clin Exp Allergy. 2013; 43, 198-211.

53. Antony KM, Ma J, Mitchell KB, et al. The preterm placental microbiome varies in association with excess maternal gestational weight gain. Am J Obstet Gynecol. 2015; 212, e1-653.

54. Rautava S, Collado MC, Salminen S, et al. Probiotics modulate host-microbe interaction in the placenta and fetal gut: a doubleblind, randomized, placebo-controlled trial. Neonatology. 2012; 102, 178-184.

55. Zi MY, Longo PL, Bueno-Silva B, Mayer MP. Mechanisms involved in the association between periodontitis and complications in pregnancy. Front Public Health. 2015; 2, 290.

56. Jiménez E, Marín ML, Martín R, et al. Is meconium from healthy newborns actually sterile? Res Microbiol. 2008; 159, 187-193.

57. Moles L, Gómez M, Heilig H, et al. Bacterial diversity in meconium of preterm neonates and evolution of their fecal microbiota during the first month of life. PLoS One. 2013; 8, e66986.

58. Ardissone AN, de la Cruz DM, Davis-Richardson AG, et al. Meconium microbiome analysis identifies bacteria correlated with premature birth. PLoS One. 2014; 9, e90784.
59. Harmsen HJ, Wildeboer-Veloo AC, Raangs GC, et al. Analysis of intestinal flora development in breast-fed and formula-fed infants by using molecular identification and detection methods. J Pediatr Gastroenterol Nutr. 2000; 30, 61-67.

60. Roger LC, Costabile A, Holland DT, Hoyles L, McCartney AL. Examination of faecal Bifidobacterium populations in breast- and formula-fed infants during the first 18 months of life. Microbiology. 2010; 156, 3329-3341.

61. Bode L. Human milk oligosaccharides: every baby needs a sugar mama. Glycobiology. 2012; 22, 1147-1162.

62. Dogra S, Sakwinska O, Soh SE, et al. Dynamics of infant gut microbiota are influenced by delivery mode and gestational duration and are associated with subsequent adiposity. MBio. 2015; 6, e02419-14.

63. Rautava S, Walker WA. Academy of Breastfeeding Medicine founder's lecture 2008: breastfeeding - an extrauterine link between mother and child. Breastfeed Med. 2009; 4, 3-10.

64. Urbaniak C, Cummins J, Brackstone M, et al. Microbiota in human breast tissue. Appl Environ Microbiol. 2014; 80, 3007-3014.

65. Cabrera-Rubio R, Collado MC, Laitinen K, et al. The human milk microbiome changes over lactation and is shaped by maternal weight and mode of delivery. Am J Clin Nutr. 2012; 96, 544-551.

66. Perez PF, Doré J, Leclerc M, et al. Bacterial imprinting of the neonatal immune system: lessons from maternal cells? Pediatrics. 2007; 119, e724-e732.

67. Abrahamsson TR, Sinkiewicz G, Jakobsson T, Fredrikson M, Björkstén B. Probiotic lactobacilli in breast milk and infant stool in relation to oral intake during the first year of life. J Pediatr Gastroenterol Nutr. 2009; 49, 349-354.

68. Grönlund MM, Gueimonde M, Laitinen K, et al. Maternal breast-milk and intestinal bifidobacteria guide the compositional development of the Bifidobacterium microbiota in infants at risk of allergic disease. Clin Exp Allergy. 2007; 37, 1764-1772.

69. Martín R, Langa S, Reviriego C, et al. Human milk is a source of lactic acid bacteria for the infant gut. J Pediatr. 2003; 143, 754-758.

70. Khodayar-Pardo P, Mira-Pascual L, Collado MC, MartínezCosta C. Impact of lactation stage, gestational age and mode of delivery on breast milk microbiota. J Perinatol. 2014; 34, 599-605.

71. González R, Maldonado A, Martín V, et al. Breast milk and gut microbiota in African mothers and infants from an area of high HIV prevalence. PLoS One. 2013; 8, e80299.

72. Yan J, Liu L, Zhu Y, Huang G, Wang PP. The association between breastfeeding and childhood obesity: a meta-analysis. BMC Public Health. 2014; 14, 1267.

73. Collado MC, Laitinen K, Salminen S, Isolauri E. Maternal weight and excessive weight gain during pregnancy modify the immunomodulatory potential of breastmilk. Pediatr Res. 2012; 72, 77-85.

74. Quigley M, McGuire W. Formula versus donor breast milk for feeding preterm or low birth weight infants. Cochrane Database Syst Rev. 2014; 4, CD002971.

75. Trend S, Strunk T, Hibbert J, et al. Antimicrobial protein and peptide concentrations and activity in human breast milk consumed by preterm infants at risk of late-onset neonatal sepsis. PLoS One. 2015; 10, e0117038. 
76. Rautava S, Nanthakumar NN, Dubert-Ferrandon A, et al. Breast milk-transforming growth factor- $\beta 2$ specifically attenuates IL-1 $\beta$-induced inflammatory responses in the immature human intestine via an SMAD6- and ERK-dependent mechanism. Neonatology. 2011; 99, 192-201.

77. Rautava S, Lu L, Nanthakumar NN, Dubert-Ferrandon A, Walker WA. TGF- $\beta 2$ induces maturation of immature human intestinal epithelial cells and inhibits inflammatory cytokine responses induced via the NF- $\mathrm{KB}$ pathway. J Pediatr Gastroenterol Nutr. 2012; 54, 630-638.

78. Lee J, Kim HS, Jung YH, et al. Oropharyngeal colostrum administration in extremely premature infants: an RCT. Pediatrics. 2015; 135, e357-e366.

79. Penttila IA, van Spriel AB, Zhang MF, et al. Transforming growth factor-beta levels in maternal milk and expression in postnatal rat duodenum and ileum. Pediatr Res. 1998; 44, 524-531.

80. Kainonen E, Rautava S, Isolauri E. Immunological programming by breast milk creates an anti-inflammatory cytokine milieu in breast-fed infants compared to formula-fed infants. Br J Nutr. 2013; 109, 1962-1970.

81. Weiner HL. Oral tolerance: immune mechanisms and the generation of Th3-type TGF-beta-secreting regulatory cells. Microbes Infect. 2001; 3, 947-954.

82. Verhasselt V, Milcent V, Cazareth J, et al. Breast milk-mediated transfer of an antigen induces tolerance and protection from allergic asthma. Nat Med. 2008; 14, 170-175.

83. Nguyen DN, Jiang P, Jacobsen S, et al. Protective effects of transforming growth factor $\beta 2$ in intestinal epithelial cells by regulation of proteins associated with stress and endotoxin responses. PLoS One. 2015; 10, e 0117608.

84. Kalliomäki M, Ouwehand A, Arvilommi H, Kero P, Isolauri E. Transforming growth factor-beta in breast milk: a potential regulator of atopic disease at an early age. J Allergy Clin Immunol. 1999; 104, 1251-1257.

85. Oddy WH, Halonen M, Martinez FD, et al. TGF-beta in human milk is associated with wheeze in infancy. J Allergy Clin Immunol. 2003; 112, 723-728.

86. Laiho K, Lampi AM, Hämäläinen $M$, et al. Breast milk fatty acids, eicosanoids, and cytokines in mothers with and without allergic disease. Pediatr Res. 2003; 53, 642-647.

87. Neu J, Walker W. Necrotizing enterocolitis. N Engl J Med. 2011; 364, 255-264.

88. Maheshwari A, Schelonka RL, Dimmitt RA, et al. Cytokines associated with necrotizing enterocolitis in extremely-low-birthweight infants. Pediatr Res. 2014; 76, 100-108.

89. Frost BL, Jilling T, Lapin B, Maheshwari A, Caplan MS. Maternal breast milk transforming growth factor-beta and feeding intolerance in preterm infants. Pediatr Res. 2014; 76, 386-393.

90. Nguyen DN, Sangild PT, Ostergaard MV, Bering SB, Chatterton DE. Transforming growth factor- $\beta 2$ and endotoxin interact to regulate homeostasis via interleukin-8 levels in the immature intestine. Am J Physiol Gastrointest Liver Physiol. 2014; 307, G689-G699.

91. Oz HS, Ray M, Chen TS, McClain CJ. Efficacy of a transforming growth factor beta 2 containing nutritional support formula in a murine model of inflammatory bowel disease. J Am Coll Nutr. 2004; 23, 220-226.

92. Rautava S, Kalliomäki M, Isolauri E. Probiotics during pregnancy and breast-feeding might confer immunomodulatory protection against atopic disease in the infant. J Allergy Clin Immunol. 2002; 109, 119-121.

93. Huurre A, Laitinen K, Rautava S, Korkeamäki M, Isolauri E. Impact of maternal atopy and probiotic supplementation during pregnancy on infant sensitization: a double-blind placebo-controlled study. Clin Exp Allergy. 2008; 38, 1342-1348.

94. Böttcher MF, Abrahamsson TR, Fredriksson M, Jakobsson T, Björkstén B. Low breast milk TGF-beta2 is induced by Lactobacillus reuteri supplementation and associates with reduced risk of sensitization during infancy. Pediatr Allergy Immunol. 2008; 19, 497-504.

95. Fujii T, Ohtsuka Y, Lee T, et al. Bifidobacterium breve enhances transforming growth factor beta1 signaling by regulating Smad7 expression in preterm infants. J Pediatr Gastroenterol Nutr. 2006; 43, 83-88.

96. Hill C, Guarner F, Reid G, et al. Expert consensus document. The International Scientific Association for Probiotics and Prebiotics consensus statement on the scope and appropriate use of the term probiotic. Nat Rev Gastroenterol Hepatol. 2014; 11, 506-514.

97. Panduru M, Panduru NM, Sălăvăstru CM, Tiplica GS. Probiotics and primary prevention of atopic dermatitis: a metaanalysis of randomized controlled studies. J Eur Acad Dermatol Venereol. 2015; 29, 232-242.

98. AlFaleh K, Anabrees J. Probiotics for prevention of necrotizing enterocolitis in preterm infants. Cochrane Database Syst Rev. 2014; 4, CD005496.

99. Abrahamsson TR, Rautava S, Moore AM, Neu J, Sherman PM. The time for a confirmative necrotizing enterocolitis probiotics prevention trial in the extremely low birth weight infant in North America is now!. J Pediatr. 2014; 165, 389-394.

100. Kalliomäki M, Salminen S, Arvilommi H, et al. Probiotics in primary prevention of atopic disease: a randomised placebocontrolled trial. Lancet. 2001; 357, 1076-1079.

101. Kalliomäki M, Salminen S, Poussa T, Arvilommi H, Isolauri E. Probiotics and prevention of atopic disease: 4-year follow-up of a randomised placebo-controlled trial. Lancet. 2003; 361, 1869-1871.

102. Kalliomäki M, Salminen S, Poussa T, Isolauri E. Probiotics during the first 7 years of life: a cumulative risk reduction of eczema in a randomized, placebo-controlled trial. J Allergy Clin Immunol. 2007; 119, 1019-1021.

103. Rinne M, Kalliomäki M, Salminen S, Isolauri E. Probiotic intervention in the first months of life: short-term effects on gastrointestinal symptoms and long-term effects on gut microbiota. J Pediatr Gastroenterol Nutr. 2006; 43, 200-205.

104. Gueimonde M, Kalliomäki M, Isolauri E, Salminen S. Probiotic intervention in neonates - will permanent colonization ensue? J Pediatr Gastroenterol Nutr. 2006; 42, 604-606.

105. Rautava S, Kainonen E, Salminen S, Isolauri E. Maternal probiotic supplementation during pregnancy and breast-feeding reduces the risk of eczema in the infant. J Allergy Clin Immunol. 2012; 130, 1355-1360.

106. Ganguli K, Collado MC, Rautava J, et al. Lactobacillus rhamnosus GG and its SpaC pilus adhesin modulate inflammatory responsiveness and TLR-related gene expression in the fetal human gut. Pediatr Res. 2015; 77, 528-535. 